\title{
Comparison of distance performances of modulation schemes in intelligent transport system image sensor communication
}

\author{
Takuya Yamamoto ${ }^{1, \text { a) }}$, Takaya Yamazato ${ }^{1}$, Hiraku Okada ${ }^{1}$, \\ Masayuki Kinoshita ${ }^{2}$, Koji Kamakura ${ }^{2}$, Shintaro Arai $^{3}$, \\ Tomohiro Yendo ${ }^{4}$, and Toshiaki Fujii ${ }^{1}$ \\ ${ }^{1}$ Nagoya University, \\ Furo-cho, Chikusa-ku, Nagoya-shi, Aichi 464-8603, Japan \\ ${ }^{2}$ Chiba Institute of Technology, \\ 2-17-1 Tsudanuma, Narashino-shi, Chiba 275-0016, Japan \\ ${ }^{3}$ Okayama University of Science, \\ 1-1 Ridai-cho, Kita-ku, Okayama-shi, Okayama 700-0005, Japan \\ ${ }^{4}$ Nagaoka University of Technology, \\ 1603-1 Kamitomioka, Nagaoka-shi, Niigata 940-2188, Japan \\ a)yamamoto.takuya@k.mbox.nagoya-u.ac.jp
}

\begin{abstract}
This study describes intelligent transport image sensor communication (ITS-ISC) systems that use LED arrays as transmitters that imitate traffic lights. Three signal-transmission schemes -luminance modulation, spatial modulation, and combined luminance and spatial modulation- use LED array. However, their suitability for ITS-ISC has not been explored yet. Therefore, we compare the communication performance of these three systems under strong saturation and present the results.
\end{abstract}

Keywords: visible light communication, image sensor communication, intelligent transport system, high-speed camera, LED array

Classification: Wireless Communication Technologies

\section{References}

[1] K. Siddiqi, A.D. Raza, and S.S. Muhammad, "Visible light communication for v2v intelligent transport system," 2016 IEEE International Conference on Broadband Communications for Next Generation Networks and Multimedia Applications (CoBCom), pp. 1-4, Sept. 2016. DOI: 10.1109/cobcom.2016.7593510

[2] P. Ji, H.-M. Tsai, C. Wang, and F. Liu, "Vehicular visible light communications with LED taillight and rolling shutter camera," 2014 IEEE 79th Vehicular Technology Conference (VTC Spring), pp. 1-6, May 2014. DOI: 10.1109/ vtcspring.2014.7023142

[3] I. Takai, S. Ito, K. Yasutomi, K. Kagawa, M. Andoh, and S. Kawahito, "LED and CMOS image sensor based optical wireless communication system for auto- 
motive applications," IEEE Photon. J., vol. 5, no. 5, 6801418, Oct. 2013. DOI: 10.1109/jphot.2013.2277881

[4] S. Kamegawa, M. Kinoshita, T. Yamazato, H. Okada, T. Fujii, K. Kamakura, T. Yendo, and S. Arai, "Performance evaluation of precoded pulse width modulation for image sensor communication," 2018 IEEE Globecom Workshops (GC Wkshps), pp. 1-7, Dec. 2018. DOI: 10.1109/glocomw.2018.8644347

[5] T. Yamamoto, et al., "A comparison of distance performances of modulation schemes in an its image sensor communication," 2020 International Conference on Emerging Technologies for Communications, ICETC2020, Dec. 2020.

[6] J. Roels, J. Aelterman, J. De Vylder, H. Luong, Y. Saeys, and W. Philips, "Bayesian deconvolution of scanning electron microscopy images using pointspread function estimation and non-local regularization," 2016 IEEE 38th Annual International Conference of the IEEE Engineering in Medicine and Biology Society (EMBC), pp. 443-447, Aug. 2016. DOI: 10.1109/embc.2016.7590735

\section{Introduction}

Intelligent transport systems (ITSs) facilitate information exchange among people, roads, and vehicles, which enable automatic-driving and driving-assistance technologies. Applications such as visual aids for automated driving, object detection, and drive recorders use image sensors. LED lights are used as transmitters in image sensor communications (ISC) in which an image sensor, as a receiver, is applied to traffic signals and vehicle tail lights [1]. Since a sensor can be used as a transmitter and receiver, ISC can be used with other ITS applications [2]. However, the image sensors used in smartphones and other devices are not suitable for mobile environments due to their slow frame rate of 30 frames per second. In this study, we consider the intelligent transport image sensor communication (ITS-ISC) systems that employ LED traffic lights and high-speed image sensors mounted in cars as transmitters and receivers, respectively [3].

Communication performance decreases as the distance between a transmitter and receiver increases since the data are received as pictures. To compensate, multiple LEDs are used in a single block to transmit one symbol. Increasing the number of transmission symbols per block increases transmission rates. In this configuration, a multilevel modulation in a spatial, luminance, or combined spatial and luminance domain [4] is a strategy to increase data transmission rates. For example, spatial multilevel schemes boost data rates by increasing the number of LEDs in one block from four to eight. In contrast, multilevel luminance systems improve data rates by increasing the brightness level in one block, from to eight four pulse width modulations (PWMs) to represent the brightness of LEDs.

However, their suitability for ITS-ISC has not been explored.

This study compares the distance performance of three modulation schemes by using a simulation. Scheme 1 is a luminance modulation, scheme 2 is a spatial modulation, and scheme 3 is a combined luminance and spatial modulation.

In [5], the tolerance of saturation was not discussed. Therefore, this study compares the distance performance under strong saturation by simulation. 


\section{System overview}

Figure 1 illustrates the ITS-ISC system model with a high-speed camera. PWM drives the transmitter LED array with 256 voltage levels; the LED brightness changes linearly with voltage levels. In an actual communication environment, LEDs are placed in a traffic signal circularly. However, in this simulation, we use a square LED array with $16 \times 16$ LEDs for simplicity and consider this array to be transmitting $k \times k$ symbols simultaneously.

One block is defined as $16 / k \times 16 / k$ LEDs. To enable communication over long distances, we consider multiple LEDs that transmit one symbol as one block.

A high-speed image sensor acts as a receiver for capturing an image of the LED array and receives optical signals. The images are captured in grayscale and assigned integer values of $0-255 /$ pixel (8-bit). The receiver detects and tracks the LED arrays that transmitted signals in the images. The received image, shown in Fig. 1, was captured using a high-speed image sensor. Then, the LED array is detected and the average brightness value of each block is processed to make a symbol decision.

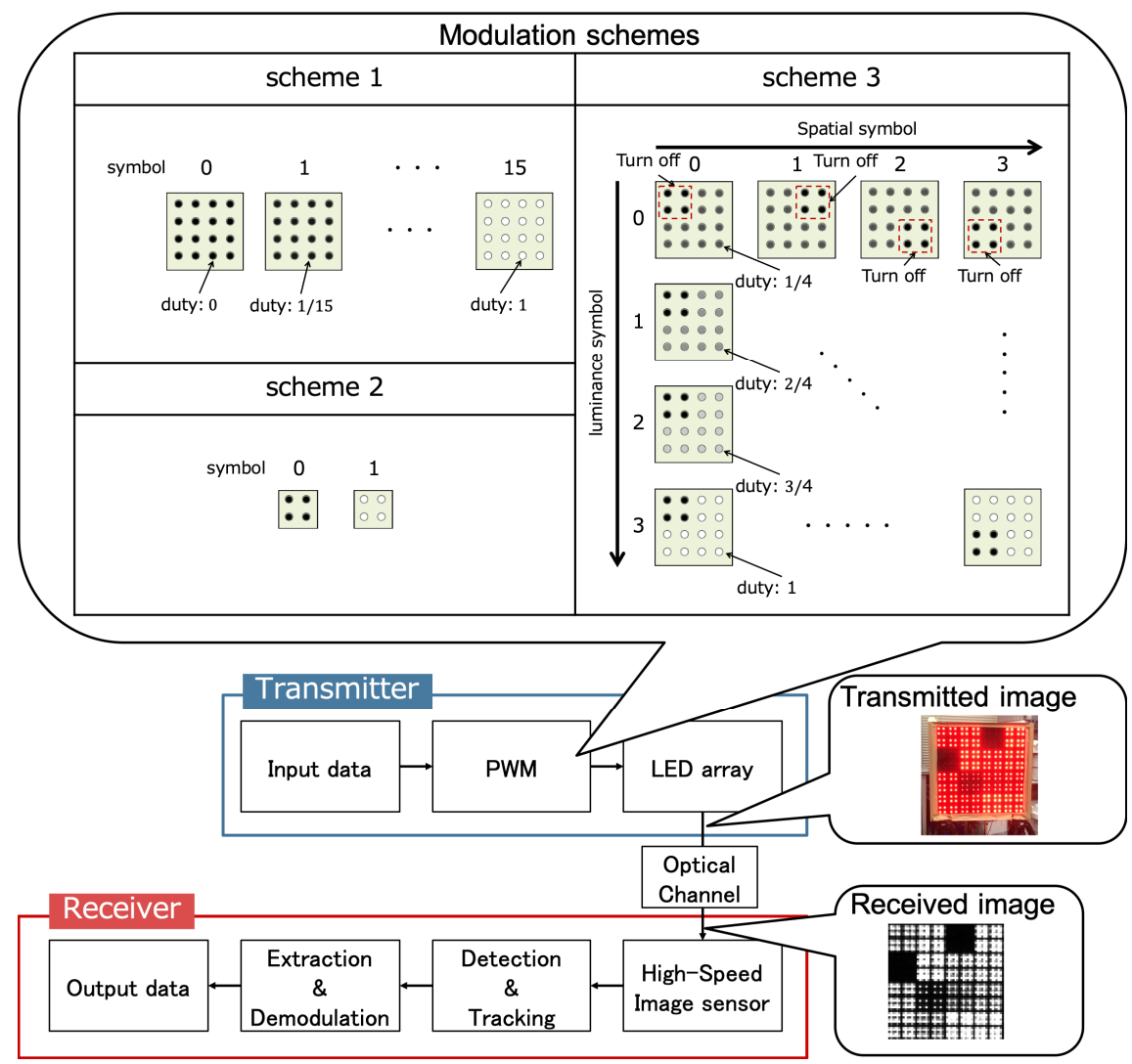

Fig. 1. System model for visible light communication using an LED array and modulation schemes compared in this study

\section{Modulation schemes using LED arrays and image sensors}

ITS-ISC communication rate can be improved by increasing the number of multilevel symbol schemes in one block. Two parameters -luminance and spatial distribution- 
can be used to add symbols in multilevel modulation schemes. In addition, we can expand the number of symbols in both parameters. Therefore, this study compares the performance of three modulation schemes: luminance only, spatial only, and combined luminance and spatial. Modulation schemes of Fig. 1 shows an example of the display patterns for each of the symbols of the three modulation schemes compared in this study. The number of transmitted bits for each modulation per captured image is the same.

Scheme 1 boosts the transmitted bits by increasing the luminance levels in one block. For example, to transmit an M-level symbol, a $\log _{2} M$-bit signal can be conveyed by increasing the applied voltage duty ratio to the $M$ level of $0,1 /(M-$ $1), 2 /(M-1), \ldots,(M-2) /(M-1), 1$ for one block of LEDs. In modulation schemes of Fig. 1, the display pattern of scheme 1 transmits 16-level (4-bit) symbols.

To expand the number of symbol levels in a space, a transmitter increases the number of blocks rather than the number of levels in the luminance. In modulation schemes of Fig. 1, $2 \times 2$ LEDs are considered as one block, so the total number of blocks in one LED pattern of scheme 2 is four times greater than that of scheme 1 .

Scheme 3 transmits a signal by turning off some LEDs in a block. In modulation schemes of Fig. 1, $2 \times 2$ LEDs from one block are turned off to multiply the number of symbols in the space so that scheme 3 turns off one of the four blocks (top-left, top-right, bottom-right, or bottom-left) and transmits four levels of spatial symbols. A block can also display a luminance symbol by maintaining the state of the block that is turned off and changing the brightness of the remaining LEDs. In scheme 3, luminance modulation adjusts at the $\mathrm{M}$ level of $1 / M, 2 / M, \ldots,(M-1) / M, 1$ without using the duty ratio of 0 . So the turned-off part can be identified. In Fig. 1, one block transmits four levels of luminance symbols.

Scheme 1 and scheme 2 must capture each block with at least $2 \times 2$ pixels from the sampling theorem. Since scheme 3 has to decide which LEDs inside the block light should be on or off, we need to sample the block with $4 \times 4$ pixels.

Note that a pilot signal must be sent as a header for demodulation when luminance modulation occurs; this is employed for the turned-off block is employed. The pilot signal transmits all luminance symbols.

\section{Comparing modulation methods using simulation}

We compare the communication performance of the three modulation schemes using a simulation that employs the same number of transmitted bits per display pattern, as shown in Fig. 1.

\subsection{Simulation setup}

The simulator creates LED array images that are captured using the image sensor and performs three processes that determine the LED array's shape, the convolution of the point spread function(PSF), and sampling.

When determining the shape of the LED array, the number and shape of LEDs and, their positions and luminance are established to generate the transmitted images. The simulator generates images with more pixels than a received image size. Here, 256 circular LEDs are allocated to equally spaced squares. 
Table I. Equipment and simulator specifications

\begin{tabular}{|c|c|}
\hline & Equipment specifications \\
\hline Transmitter & HW-LEDARRAY2 \\
\hline Transmitter size & $46 \times 46 \mathrm{~cm}^{2}$ \\
\hline Frequency & $0.5[\mathrm{kHz}]$ \\
\hline Receiver & Photron IDP-Express R2000-F made by photron \\
\hline Focus length & $35[\mathrm{~mm}]$ \\
\hline \multicolumn{2}{|c|}{ Simulator specifications } \\
\hline LED array base image size & $400 \times 400$ pixel \\
\hline PSF size & $401 \times 401$ pixel \\
\hline PSF variance $\sigma^{2}$ & 10 \\
\hline Transmitted bit number & 6208 bit \\
\hline F-numbers & 16 \\
\hline
\end{tabular}

The PSF is widely used in imaging techniques, such as image restoration. It represents the diffusion of light from the point source [6]. In the simulation, the PSF is defined by the two-dimensional Gaussian function and the gain $G(F)$, which varies with the F-number, as

$$
\operatorname{PSF}(x, y, \sigma, F)=\left(\frac{1.04}{F^{2}}+\frac{0.04}{F}-0.001\right) \exp \left(-\frac{x^{2}+y^{2}}{2 \sigma^{2}}\right)
$$

where $x$ and $y$ denote the distance from the point source, and $\sigma^{2}$ denotes the degree of diffusion that depends on the focus. The F-number is defined as the diameter of the aperture divided by the focal length of the camera, so the maximum PSF is inversely proportional to the square of the F-number. Table I lists the equipment specifications used to derive (1). The simulator performs a convolution of (1) into the transmitted image of the LED array.

The sampling process is imitated by reducing the number of pixels from the transmitted image by an area average method in which an image resizing algorithm transforms the average of pixel values within an area of one pixel after resizing it to a new pixel value. The number of pixels to be resized is inversely proportional to the distance between the LED array and camera. The relationship between the distance and number of pixels per side of the LED array is shown below.

$$
S(d)=\operatorname{ceil}\left(\frac{1673}{d}-0.467\right)
$$

where $d$ denotes the distance between the LED array and camera, and ceil $(\cdot)$ denotes the ceiling function. Table I lists the equipment specifications used to derive (2).

We compare the performance changes in the distance of each modulation scheme by using the simulator shown. We assume perfect synchronization between the transmitter and the receiver. We measure the number of correctly received bits over the range of 20-258 m with $1 \mathrm{~m}$ as step sizes. Note that when $d<45 \mathrm{~m}$, the pixel size of the received image does not change for each measurement point. Therefore, for distances greater than $45 \mathrm{~m}$, we measure spaces at intervals corresponding to changes that are more than one pixel. 
We demodulate data from the received images generated using the simulator. In a real environment, a receiver would detect and track LED arrays from the captured images. However, these processes are not performed so the simulator only generates images of the LED arrays.

\subsection{Simulation results}

Figure 2 shows the simulation results. Note that 6,208 bits were transmitted in this simulation and the number of pixels given in the figure is the number of received pixels at the capturing point.

Scheme 1 has the worst performance due to interference with neighboring blocks. Light diffused from neighboring blocks causes varying luminance in the receiving blocks, thus decreasing performance.

Scheme 2 achieved the best performance. For scheme 2, we can achieve almost error-free communication up to $108 \mathrm{~m}$, where we can sample one block by $2 \times 2$. This result shows that scheme 2 can communicate stably up to the Nyquist rate and that the lower bound of the sampling rate can reproduce the signal. In this case, the sampling number when sampling at $2 \times 2$ pixels per block is equal to the Nyquist rate.

Scheme 3 performs better than scheme 1 but is degraded by the luminance symbols, since they require more pixels.

This simulation has stronger saturation than the simulation in [5]. Since luminance symbols are significantly affected by saturation effects, the performance of scheme 1 and scheme 3 is degraded from the results in [5]. Since scheme 2 is not affected by saturation, it is able to maintain its performance unaffected by saturation.

Therefore, luminance modulation is not suitable for communication over long distances, since it is sensitive to interference from neighboring blocks and sampling artifacts. Thus, in ITS-ISC, we recommend increasing the number of transmitted bits by expanding the number of blocks in scheme 2 .

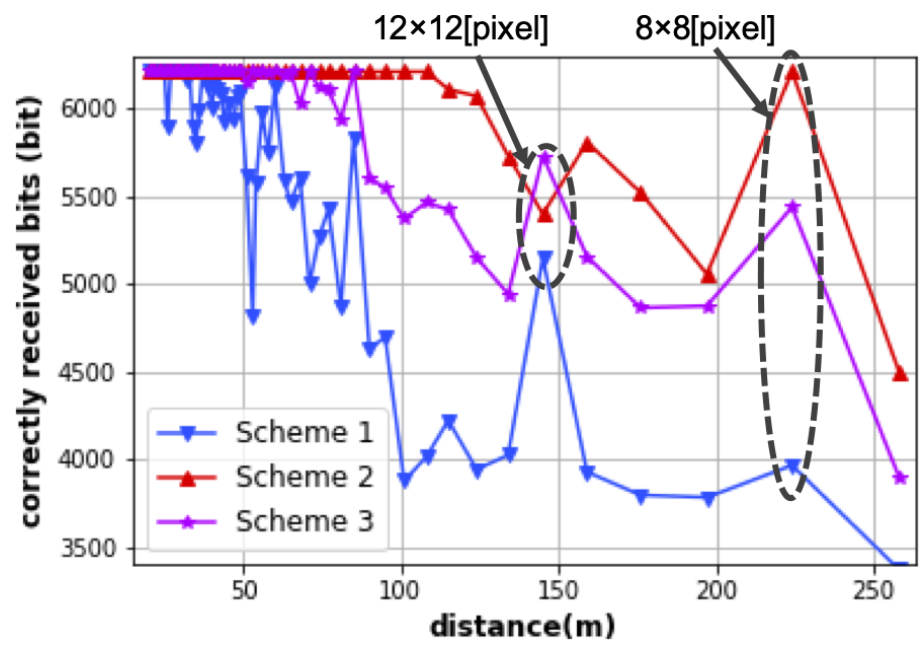

Fig. 2. Simulation results showing relation between communication distance and the number of correctly received bits 


\section{Conclusions}

In this study, we measured the performance change with distances of the luminance and spatial modulation of ITS-ISC using a simulation and compared the results. Scheme 1 increases the number of symbols in luminance, scheme 2 boosts the number of symbols spatially, and scheme 3 expands the number of symbols both spatially and in luminance. The simulation results show that an exclusively spatial modulation of scheme 2 achieves better communication performance and has tolerance to saturation than the modulation schemes by incorporating luminance modulation. Therefore, it is the most suitable for long-distance communication and should be used without luminance modulation for ITS-ISC.

\section{Acknowledgments}

The authors would like to thank Prof. Masaaki KATAYAMA of Nagoya University and Assistant Prof. BEN NAILA Chedlia of Nagoya University for their valuable suggestions. 